\title{
O efeito de um programa de exercício físico aeróbio combinado com exercícios de resistência muscular localizada na melhora da circulação sistêmica e local: um estudo de caso
}

\author{
Ricardo Bosco ${ }^{1}$, Amanda Demarchi ${ }^{1}$, Fabiana Pereira Vecchio Rebelo $^{3}$ e Tales de Carvalho ${ }^{2,3}$
}

\section{RESUMO}

Introdução: A trombofilia, estado que predispõe à ocorrência de trombose, decorre da existência de alterações da hemostasia, podendo ser congênita ou adquirida. A deficiência de proteína $S$, que ocorre pela falta do co-fator para a proteína $\mathrm{C}$, é uma alteração congênita que pode resultar em trombofilia. A reabilitação física, considerada uma terapêutica eficaz, tem seu papel definido na prevenção e tratamento de doenças cardiovasculares. Objetivo: Este estudo se propôs a verificar a influência do exercício físico aeróbio associado aos exercícios de resistência muscular localizada na circulação sistêmica e colateral do membro superior e inferior direito e na redução das conseqüências causadas pelas retrações decorrentes das cirurgias. Material e método: A amostra foi composta por uma paciente do sexo feminino, 19 anos, portadora da síndrome de hipercoagulabilidade (trombofilia) congênita por deficiência da proteína $\mathrm{S}$, com obstrução total na artéria axilar direita e parcial na artéria femoral superficial direita. O programa de exercícios foi dividido em duas fases. Fase I: Atividades para aumento da amplitude de movimentos, em que se realizavam aplicação de calor, alongamentos passivos manuais mantidos, drenagem linfática e manipulações Effleurage. Fase II: Constituída de exercício aeróbio (45 minutos de caminhada, três vezes por semana, intensidade de $60 \%$ a $85 \%$ da freqüência cardíaca máxima) e exercícios

\footnotetext{
1. Acadêmicos do curso de fisioterapia da Universidade do Estado de Santa Catarina - CEFID/UDESC

2. Professor da Universidade do Estado de Santa Catarina - CEFID/UDESC.

3. Clínica CARDIOSPORT de Prevenção e Reabilitação Cardíaca - Florianópolis.
}

Recebido em 12/1/04

Aceito em 21/1/04

Endereço para correspondência:

Ricardo Bosco

Rua Tenente Silveira 324, apto. 802

88010-301 - Florianópolis, SC, Brasil

E-mail: boscofisio@udesc.br de resistência muscular localizada (contrações dinâmicas, três séries com o máximo de repetições possíveis, baixa intensidade, favorecendo fatores aeróbios). Resultados: 1 . Melhora da circulação colateral à artéria axilar, constatada pelo aumento na velocidade de pulso sistólico (verificado através do exame doppler pulsado) - na artéria radial, passou de $3,4 \mathrm{~cm} / \mathrm{s}$ para $16,8 \mathrm{~cm} / \mathrm{s}$; na artéria braquial, de $8,7 \mathrm{~cm} /$ $\mathrm{s}$ para $45,9 \mathrm{~cm} / \mathrm{s}$. 2. Melhor tolerância ao exercício aeróbio. 3. Recuperação da capacidade funcional e amplitude de movimento do ombro. 4. Recuperação plena da autonomia para as atividades diárias. Conclusões: Os resultados obtidos demonstraram que a terapêutica adotada foi eficaz no tratamento dos transtornos provocados pela trombofilia.

Palavras-chave: Programa de reabilitação. Circulação colateral e sistêmica. Trombose. Exercícios físicos.

\section{RESUMEN}

El efecto de un programa de ejercício físico aeróbico combinado con los ejercicios de resistencia muscular localizada en la mejora de la circulacion sistemica y local. Estudio de un caso

Introdución: La trombofilia resulta de la existencia de alteraciones de la hemóstasis, pudiendo ser congénita o adquirida. La deficiencia de proteína S ocurre por la falta del cofactor para la proteína $C$, y una alteración congénita que puede resultar en trombofilia. La reabilitación física, considerada una terapéutica eficaz, tiene su papel definido en la prevención y el tratamiento de enfermedades cardiovasculares. Objetivo: En este estudio se propuso verificar la influencia del ejercicio físico aeróbico asociado a los ejercicios de resistencia muscular localizada en la circulación sistémica y colateral del miembro superior e inferior derecho y en la redución de las consecuencias causadas por las retraciones resultantes de las cirugias. Material y método: La muestra fué compuesta por una paciente de sexo femenino, de 19 años, portadora del síndrome de hipercoagulabilidad (trombofilia) congénita por deficiencia de proteína $S$, con obstrucción total de la arteria 
axilar derecha y parcial de la arteria femoral superficial derecha. El programa de ejercicios fué dividido en dos fases. Fase I: Actividades para el aumento de la amplitud de movimientos, realizada mediante la aplicación de calor, elongamientos pasivos manuales mantenidos, drenage linfático y manipulaciones Effleurage. Fase II: Constituída de ejercicio aeróbico (45 minutos de caminata, 3 veces por semana, intensidad de $60 \%$ a $85 \%$ de la frecuencia cardíaca máxima), y ejercicios de resistencia muscular localizada (contraciones dinámicas, tres series con el máximo de repeticiones posibles, baja intensidad, favoreciendo factores aeróbicos). Resultados: 1. Mejora de la circulación colateral de la arteria axilar, constatada por el aumento de la velocidad de pulso sistólico (verificado a través del examen doppler pulsado) - en la arteria radial paso de $3,4 \mathrm{~cm} / \mathrm{s}$ a $16,8 \mathrm{~cm} / \mathrm{s}$; en la arteria braquial, de $8,7 \mathrm{~cm} / \mathrm{s}$ a $45,9 \mathrm{~cm} / \mathrm{s}$. 2. Mejor tolerancia al ejercicio aeróbico. 3. Recuperación de la capacidad funcional y amplitud del movimiento del hombro. 4. Recuperación plena de la autonomía para las actividades diárias. Conclusiones: Los resultados obtenidos demostraron que la terapéutica adoptada fué eficaz en el tratamiento de los transtornos provocados por la trombofilia.

Palabras clave: Programa de rehabilitación. Circulación colateral y sistémica. Trombosis. Ejercicios físicos.

\section{INTRODUÇÃO}

A trombofilia é o estado que predispõe à ocorrência de trombose e decorre da existência de alterações da hemostasia $^{1}$. As alterações podem ser congênitas, determinadas por modificações genéticas e herdadas pelos membros da família, ou por situações adquiridas que alteram o equilíbrio da hemostasia ${ }^{2}$.

As desordens trombofílicas herdadas, como a resistência a proteínas $\mathrm{C}$ ativadas (fator $\mathrm{V}$ Leiden), deficiência da proteína $\mathrm{C}$, de proteína $\mathrm{S}$ e de antitrombina III, são anormalidades relacionadas à qualidade dos fatores ativados ${ }^{3}$. $\mathrm{O}$ mecanismo da deficiência de proteína $\mathrm{S}$ é a falta do cofator para a proteína $\mathrm{C}$, não havendo inativação dos fatores Va e do VIIIa, como ocorre na deficiência da proteína $\mathrm{C}^{2}$. A deficiência da proteína $S$ representa 5-6\% dos casos de trombofilia hereditária ${ }^{3}$.

A reabilitação cardiovascular, atualmente, vem sendo muito empregada e defendida pela comunidade médica e científica. Uma vez que o exercício físico tem seu papel claramente definido na prevenção primária e secundária, não só das doenças cardiovasculares como também de outras, ou seja, a prescrição de exercícios físicos é uma terapêutica ${ }^{4-7}$.
O ganho na amplitude do movimento é administrado para que haja mobilidade e flexibilidade dos tecidos moles que circundam a articulação (músculos, tecidos conectivos e pele $)^{9}$, como também revertendo o estado de contratura existente, devolvendo à articulação a sua amplitude normal de movimento 9,10 .

O exercício físico tem efeito benéfico que parece resultar de interações complexas de efeitos psicológicos e fisiológicos. Além disso, é adequado salientar a diminuição do estresse, a melhora da função cardiorrespiratória, a remoção de fatores como o tabagismo e a reeducação alimen$\operatorname{tar}^{5,7,11}$.

A melhora da circulação sistêmica ocorre, entre outras razões, pelo fato de o exercício contribuir para a redução do hematócrito (ao aumentar a volemia) e ocasionar elevação da plasticidade do eritrócito, promovendo acréscimo do fluxo sanguíneo e melhor distribuição do oxigênio na interface célula-capilar ${ }^{12}$. Podem, ainda, ocorrer modificações significativas tanto anatômicas como fisiológicas do sistema cardiovascular, com aprimoramento do sistema de transporte, extração e utilização do oxigênio ${ }^{13}$.

Estudos demonstram que os exercícios de resistência muscular localizada podem contribuir para o aumento da circulação sanguínea colateral ${ }^{14-17}$. Apesar disso, a teoria de que exercícios regulares promovem o desenvolvimento das artérias colaterais em seres humanos exige comprovação mais conclusiva ${ }^{13}$. Estudos em animais que tiveram suas artérias coronárias bloqueadas artificialmente e foram sujeitos a exercício regular controlado demonstraram que a vascularização coronária melhorou ${ }^{15}$, o que levou à conclusão de que o estreitamento arterial moderado e grave resulta em desenvolvimento de artérias colaterais proporcionais ao grau de obstrução ${ }^{14}$.

O propósito deste estudo foi verificar a influência do exercício físico aeróbio combinado com os exercícios de resistência muscular localizada na melhora da circulação sistêmica e local em uma paciente do sexo feminino, portadora de trombofilia por deficiência da proteína $\mathrm{S}$, com obstrução tromboembólica total na artéria axilar direita e obstrução parcial na artéria femoral superficial direita.

\section{MATERIAIS E MÉTODOS}

Esta pesquisa é um estudo de caso singular, de uma paciente portadora da síndrome de hipercoagulabilidade (trombofilia) congênita por deficiência da proteína $S$, de cor branca, sexo feminino, com 19 anos de idade, procedente de Florianópolis, previamente submetida a três angioplastias na artéria axilar direita, com foco de retrombose com obstrução total de um centímetro na artéria axilar direita e obstrução parcial na artéria femoral superficial direita. 
O tempo decorrido entre as cirurgias e o início da reabilitação física foi de sete dias, sendo que a paciente realizou tratamento clínico com anticoagulante heparina sódica (aplicação de 12/12 horas, dose de 15.000U diárias, pela via subcutânea) durante os sete primeiros dias da reabilitação e gradativamente foram administrados anticoagulantes orais, derivados cumarínicos (warfarin) com ácido fólico, os quais foram mantidos até o final da terapêutica.

Os dados foram obtidos através de entrevistas, método que pode ser utilizado quando se pretende complementar dados extraídos de outras fontes, ou quando não há fontes mais seguras para obtenção das informações desejadas. Outras maneiras de obter os dados foram a mensuração da frequiência cardíaca no momento da claudicação do membro inferior direito e a distância percorrida no período de 30 minutos.

A realização do exame complementar Eco Doppler arterial colorido do membro superior direito, para verificar a fluxometria a cores, a velocidade de pico sistólica e diastólica e a presença de estenoses, na pré e pós-terapêutica, fez-se necessária para verificar a eficiência do tratamento e é uma intervenção não invasiva bastante eficaz.

Os materiais utilizados durante a terapêutica foram colchonetes, toalhas, materiais elásticos para os exercícios resistidos marca Mercur, modelo Thera-band, monitor de frequiência cardíaca da marca Polar modelo A1, estetoscópio, esfigmomanômetro da Glicomed modelo Premium e cronômetro Casio.

\section{Programa de reabilitação}

O programa de reabilitação foi executado pelo período de 15 semanas, com três sessões semanais, podendo ser dividido em duas fases:

\section{Atividades para aumentar a amplitude de movimento}

- Esta fase teve duração de quatro semanas e ocorreu por ter a paciente sido submetida a três angioplastias (duas com incisão na fossa ulnar direita e uma na região axilar), o que fez com que as articulações do ombro e cotovelo direitos ficassem em contratura devido às adesões cicatriciais, resultando em limitação na mobilidade articular.

Nesta fase a sessão era iniciada por prévia aplicação de calor na área ao redor das articulações a serem alongadas, uma vez que o aquecimento do tecido mole e músculos antes do alongamento aumenta a extensibilidade do tecido encurtado ${ }^{8,10,18}$. Dando sequiência à sessão, eram realizados alongamentos passivos manuais mantidos, nos quais o terapeuta aplica uma força externa e controla a direção, velocidade, intensidade e duração do alongamento (em media quatro repetições de 30 segundos) ${ }^{17}$.

Para aplicação das técnicas de alongamento foram considerados os planos anatômicos e funcionais de movimen- to, sendo que no ombro foi dada ênfase ao aumento da flexão, hiperextensão, abdução, rotação externa, rotação interna e abdução horizontal e, para o cotovelo, extensão, supinação e pronação ${ }^{10,19}$.

$\mathrm{Na}$ sequiência dos alongamentos, realizava-se a drenagem linfática do membro para solução do linfedema e manipulações de Effleurage através de movimentos de alisamento lento, realizado com pressão crescente, na direção do fluxo venoso e linfático, para alívio da dor ${ }^{20}$.

\section{Exercícios aeróbios e de resistência muscular loca-} lizada - Esta fase teve a duração de 11 semanas e procurou verificar se esta modalidade terapêutica promoveria melhor fluxo sanguíneo nos membros afetados através do aprimoramento da circulação colateral à artéria axilar direita. A sessão, nesta fase, era composta de dois períodos:

\subsection{Exercício aeróbio}

O exercício aeróbio foi realizado três vezes por semana, com cada período durando 45 minutos, dos quais os sete primeiros minutos eram utilizados para o aquecimento: três minutos destinados para alongar os principais grupos musculares a fim de reduzir a probabilidade de lesar um músculo ou tecido conjuntivo; quatro minutos de caminhada em baixa intensidade recomendados para elevar a temperatura muscular e acelerar o fluxo sanguíneo ${ }^{4,21}$.

O treinamento aeróbio foi realizado por 30 minutos, com a freqüência cardíaca-alvo estabelecida entre $60 \%$ a $85 \%$ da freqüência cardíaca máxima observada em um teste convencional de esforço ${ }^{11,22-24}$. Dentro desses valores tentavase manter a paciente em atividade, mesmo diante dos sintomas/sinais de claudicação e desconforto no membro inferior direito, tendo por finalidade aprimorar a circulação colateral da artéria femoral superficial ${ }^{23,25}$.

Os exercícios de volta à calma (esfriamento) eram realizados por oito minutos; cinco minutos de caminhada em baixa intensidade, utilizada para prevenir a estagnação do sangue nas extremidades, particularmente nas pernas; três minutos de alongamentos estáticos para prevenir o aparecimento de lesões musculares ${ }^{4,5,21}$.

\subsection{Exercícios de resistência muscular localizada}

Os exercícios de resistência muscular localizada foram efetuados na sequiência do exercício aeróbio ${ }^{5}$ e constituíamse de contrações dinâmicas realizadas em três séries com o máximo de repetições possíveis, sem ou com baixa resistência, intensidade baixa e longa duração predominando o fator aeróbio, pois não visavam a hipertrofia muscular, mas sim o aumento no fluxo sanguíneo local e uma melhora ou neoformação vascular ${ }^{5,17}$.

No programa de exercícios de resistência as características anatômicas, cinesiológicas e funcionais próprias ao 
TABELA 1

Evolução semanal das atividades realizadas pela paciente, observada a possibilidade plena de execução

\begin{tabular}{|c|c|c|c|c|}
\hline Atividades & $\begin{array}{l}\text { 1a semana } \\
\text { (início) }\end{array}$ & $\begin{array}{c}2^{a} \text { a } 5^{a} \\
\text { semana }\end{array}$ & $\begin{array}{c}\text { 6a a 9a } \\
\text { semana }\end{array}$ & $\begin{array}{l}10^{a} \text { a } 15^{a} \\
\text { semana }\end{array}$ \\
\hline Escrever & Impossibilitado & 3 linhas & 30 linhas & Sem restrições \\
\hline Banhar-se & Com auxílio** & Região do tronco & Região do tronco MMSS*** & Sem restrição \\
\hline Escovar dentes & Com auxílio** & MMSS*** apoiados & MMSS*** apoiados & Sem restrições \\
\hline Trocar de roupa & Com auxílio** & Roupas largas & Abotoar blusa & Sem restrições \\
\hline Alimentação & Com auxílio** & Com restrições & Com restrições & Sem restrições \\
\hline Trabalhos domiciliares* & Não realizava & Não realizava & Recolhia a louça & Lavava a louça \\
\hline $\begin{array}{l}\text { * Recolher e lavar a louça. } \\
\text { ** Auxílio prestado por familiares } \\
\text { *** Membros superiores. }\end{array}$ & & & & \\
\hline
\end{tabular}

membro superior foram levadas em consideração, assim como a limitação funcional imposta pelo problema ${ }^{10,19}$. As técnicas utilizadas foram: abdução horizontal do ombro, rotação interna e externa, flexão e extensão do cotovelo, flexão do punho e flexão e extensão dos dedos e polegares $^{8,19}$.

Ao término desse período eram realizados alongamentos, bem como relaxamento muscular através de massagem, prevenindo lesões musculares e a estagnação sanguínea ${ }^{4,5}$.

Vale salientar que ao início e ao final de cada sessão eram mensuradas a pressão arterial diastólica e sistólica, a freqüência cardíaca, a freqüência respiratória e a presença do pulso radial direito e esquerdo.

\section{RESULTADOS E DISCUSSÕES}

$\mathrm{O}$ retorno à vida produtiva foi um dos resultados da terapêutica aplicada à paciente. Dados marcantes foram relatados, tais como o simples ato de puxar a coberta à noite com o membro superior direito, as atividades escolares (ato de escrever e levar o material), os hábitos de higiene, a alimentação e o ato de trocar de roupa, impraticáveis ao início da terapêutica, passaram gradativamente a ser efetuados e ao termino do tratamento não se constatavam limitações (tabela 1). A reabilitação possibilitou uma melhora na qualidade de vida através da evolução na capacidade funcional promovendo maior independência nas atividades de vida diárias ${ }^{5,11,19}$.

\section{Exercícios aeróbios}

O gráfico 1 apresenta o aumento gradual da distância percorrida durante os 30 minutos de treinamento aeróbio, que ao início do tratamento era de 2.400 metros e, ao final, de 4.000 metros, um aumento de $66,6 \%$ em relação à distancia inicial.
No gráfico 2, pode-se observar que a frequiência cardíaca que a paciente possuía no momento da claudicação aumentou de 108, no início da terapêutica, para 146 batimentos por minuto ao término, o que indica um acréscimo da intensidade para se levar a paciente à claudicação. Ressalta-se que durante os 30 minutos de treino aeróbio (caminhada), levava-se a paciente a claudicar duas vezes o membro inferior direito.

É interessante observar que a evolução da distância percorrida coincide com o aumento da freqüência cardíaca que

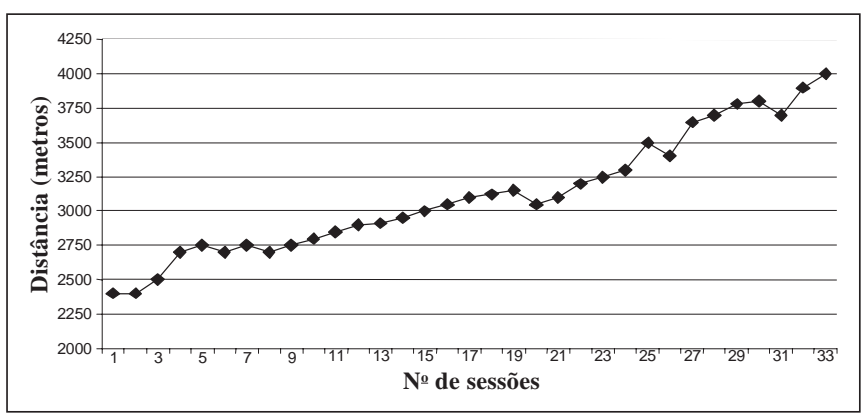

Gráfico 1 - Distância percorrida em cada sessão no tempo de 30 minutos de treinamento aeróbio

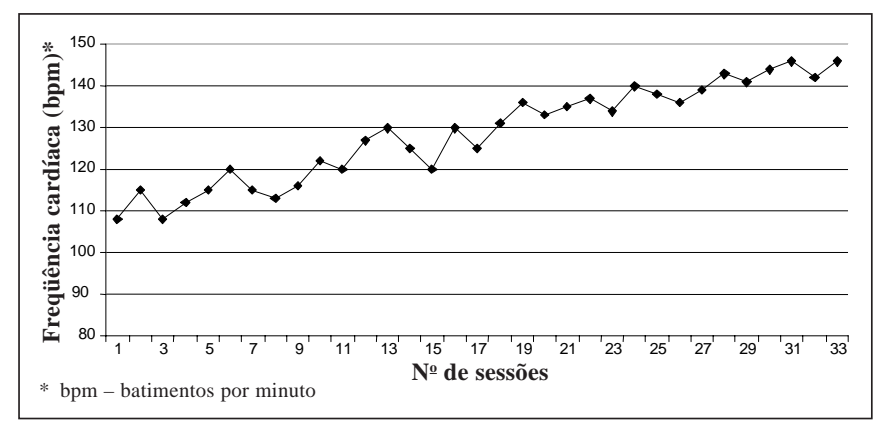

Gráfico 2 - Freqüência cardíaca no momento da claudicação, verificada em cada sessão 
TABELA 2

Evolução mensal dos exercícios de resistência muscular localizada observando o número de repetições e a carga

\begin{tabular}{|c|c|c|c|c|}
\hline Grupo muscular & $\begin{array}{c}\text { № de repetições } \\
\text { e carga } \\
\text { início }\end{array}$ & $\begin{array}{c}\text { № de repetições } \\
\text { e carga } \\
\text { 1o mês }\end{array}$ & $\begin{array}{c}\text { № de repetições } \\
\text { e carga } \\
\text { 2o mês }\end{array}$ & $\begin{array}{c}\text { № de repetições } \\
\text { e carga } \\
\text { 3 mês }\end{array}$ \\
\hline Ad. horizontal & $3 \times 8 /$ Sem & $3 \times 12 / \mathrm{Sem}$ & $3 \times 15 /$ Sem & $3 \times 19 / \mathrm{Sem}$ \\
\hline Rot. interna & $3 \times 10 /$ Leve* & $3 \times 15 /$ Leve* & $3 \times 20 /$ Leve* & $3 \times$ 18/Médio* \\
\hline Rot. externa & $3 \times 10 /$ Leve* & $3 \times 15 /$ Leve* & $3 \times 18 /$ Leve* $^{*}$ & $3 \times 18 /$ Médio* \\
\hline Flexão cotovelo & $3 \times 10 / \mathrm{Sem}$ & $3 \times 20 / \mathrm{Sem}$ & $3 \times 18 /$ Leve* & $3 \times 20 /$ Leve* \\
\hline Extensão cotovelo & $3 \times 10 /$ Sem & $3 \times 15 /$ Sem & $3 \times 18 /$ Sem & $3 \times 20 /$ Leve* \\
\hline Flexão punho & $3 \times 10 /$ Sem & $3 \times 15 / \mathrm{Sem}$ & $3 \times 20 /$ Leve* & $3 \times 20 /$ Leve* \\
\hline Artelhos & $3 \times 10 / \mathrm{Sem}$ & $3 \times 12 / M$ édio* & $3 \times$ 20/Médio* & $3 \times$ 20/Médio* \\
\hline
\end{tabular}

* De acordo com o fabricante Mercur.

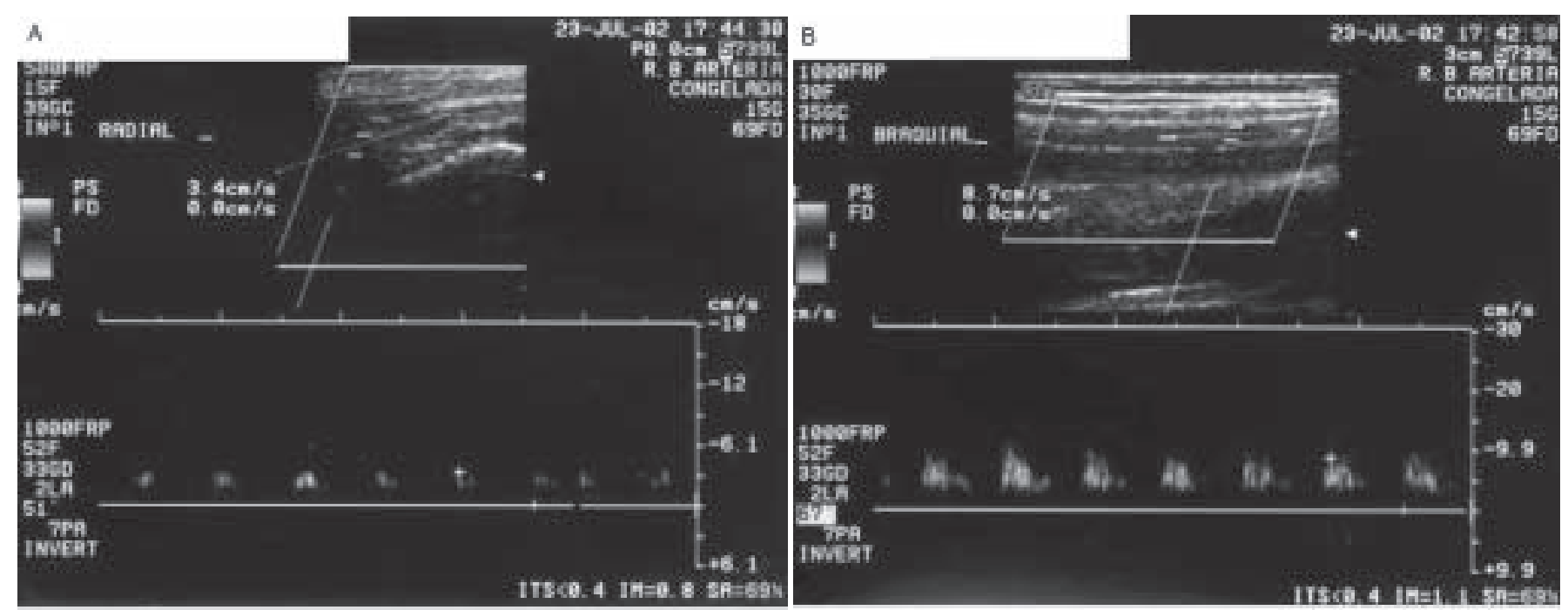

Fig. 1 -A) (radial). B) (braquial) - Doppler pulsado realizado com equipamento GE Logic 700 duas semanas antes do início da terapêutica

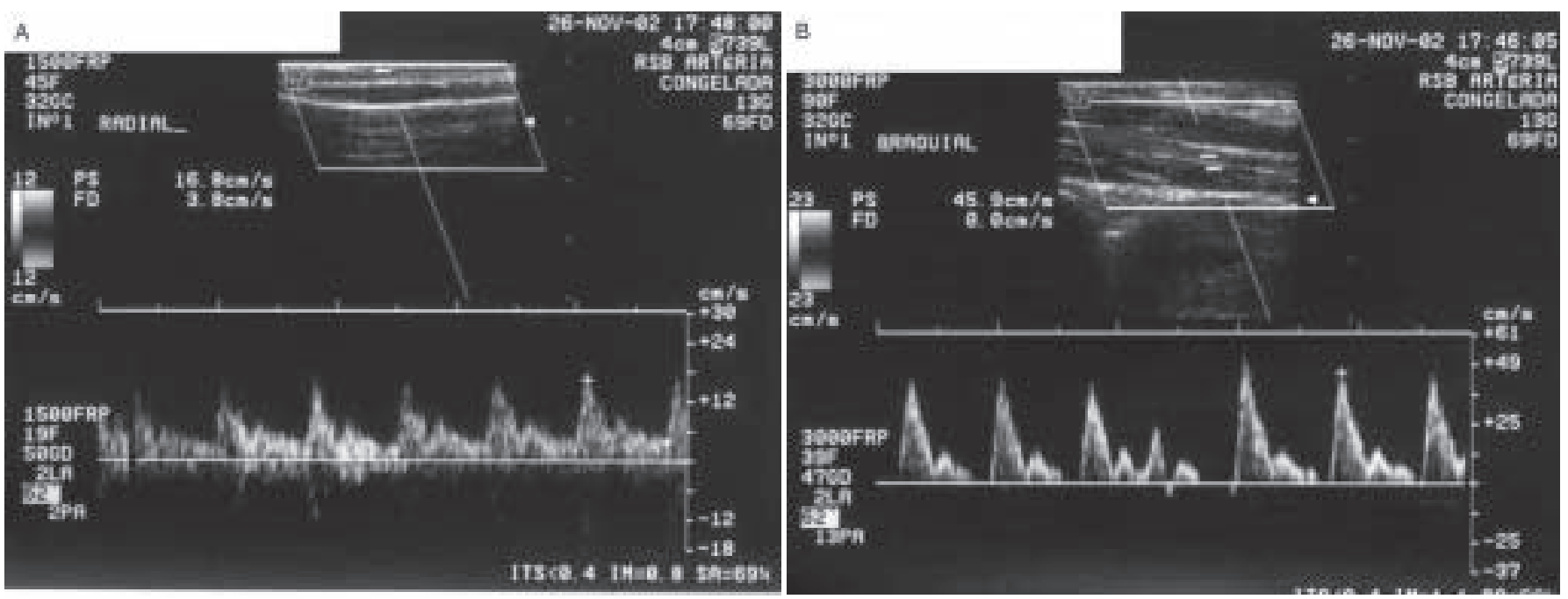

Fig. 2 - A) (radial). B) (braquial) - Doppler pulsado realizado com equipamento GE Logic 700 após o término do tratamento 
levava à claudicação, o que comprova que a tolerância ao treinamento aeróbio melhorou; estudos recentes demonstram que a caminhada pode aumentar consideravelmente a distância percorrida $(150 \%)^{26}$ ou um incremento de $122 \%$ na distância ${ }^{24}$, em pessoas que sentem claudicações nos membros inferiores por obstruções arteriais. Associa-se este aumento na capacidade de caminhar com mudanças significativas na distância e velocidade ${ }^{11}$, ou ao funcionamento do membro livre de dor ${ }^{11,24}$; relaciona-se tal evolução à regressão da obstrução parcial da artéria femoral superficial $^{27,28} \mathrm{e}$ à melhora da circulação colateral ${ }^{14,16,24}$.

\section{Exercícios de resistência muscular localizada}

A tabela 2 demonstra os grupos musculares trabalhados, o numero de repetições e a carga no decorrer da $2^{\mathrm{a}}$ fase do tratamento empregado. Observa-se que ao início da terapêutica o membro superior direito apresentava-se cianótico e gélido, necessitando recobri-lo com uma faixa feita de algodão, com intenção de o manter a certa temperatura. Com a progressão do tratamento a faixa foi sendo abandonada, pois o membro melhorou sua coloração e temperatura, ficando com aparência normal a partir da nona semana de tratamento. Pesquisas comprovam que o exercício com predominância aeróbia melhora a circulação sanguínea do membro que está sendo exercitado ${ }^{11,17}$, bem como o metabolismo músculo-esquelético, facilitando a utilização do oxigênio ${ }^{29}$.

As figuras 1 e 2 ilustram a velocidade de pulso pré e pós-terapêutica (verificado através de doppler pulsado). Pode-se observar que a melhora na velocidade de pulso sistólico ocorreu significativamente. A velocidade de pulso na artéria braquial direita, que era de $8,7 \mathrm{~cm} / \mathrm{s}$ antes da terapêutica (figura 1B), passou a $45,9 \mathrm{~cm} / \mathrm{s}$ ao termino do tra-

\section{REFERÊNCIAS}

1. Lourenço DM. Trombofilia. In: Pitta GBB, Castro AA, Buriham E, editores. Angioplastia e cirurgia vascular: guia ilustrado. Maceió: UNCISAL/ECMAL, 2000;1-9.

2. Orúe MT. Thrombophilia and stroke. Anales Sis San Navarra 2000;23:3946.

3. Hambleton J, Leung L, Levi M. Coagulation: consultative hemostasis. American Society of Hematology 2002;335-53.

4. American College of Sports Medicine. Exercise for patients with coronary artery disease. Med Sci Sports Exerc 1994;26:i-v.

5. Benetti M, Nahas M. Alterações na qualidade de vida em coronariopatas acometidos de infarto agudo do miocárdio, submetidos a diferentes tipos de tratamentos. Rev Bras Atividade Física e Saúde 2000;6:27-33.

6. Stadler E, Leite N. Reabilitação cardiovascular, custo-benefício. Rev Bras Med Esporte 2000;6:96-106.

7. Carvalho T. Reabilitação cardíaca em portadores de cardiopatia isquêmica com obstruções coronárias passíveis de tratamento intervencionista. São tamento (figura 2B), demonstrando acréscimo de 427,9\%. $\mathrm{O}$ mesmo efeito ocorreu com a velocidade de pulso da artéria radial direita, que passou de $3,4 \mathrm{~cm} / \mathrm{s}$ (figura $1 \mathrm{~A}$ ) para $16,8 \mathrm{~cm} / \mathrm{s}$ (figura $2 \mathrm{~A}$ ) de pulso sistólico, o que evidencia melhora de 394,12\%. Esse aumento na velocidade de pulso sistólico pode ocorrer por um potencial favorável da atividade fibrinolítica, em decorrência do treinamento com predominância aeróbia ${ }^{14,27,28}$, associado a uma modificação no fluxo sanguíneo da circulação colateral ${ }^{11,16}$, ou a uma vasodilatação como conseqüência do tratamento ${ }^{14}$; comprova-se, dessa forma, que o tratamento empregado não se mostra inferior aos procedimentos terapêuticos intervencionistas empregados em casos semelhantes ${ }^{7}$.

\section{CONCLUSÃO}

Através da terapêutica empregada foi observada nítida melhora da qualidade de vida da paciente, com o retorno à vida produtiva e maior independência nas atividades de vida diárias, como o ato de escrever e os hábitos de higiene.

Constatou-se, também, acentuada redução da limitação causada pelo distúrbio circulatório, verificada pelo aumento do limiar de claudicação do membro inferior direito, isto é, maior tolerância ao exercício aeróbio, e pelo aumento da velocidade de pulso sistólico na artéria braquial e radial do membro superior direito.

Este estudo sugere que o tratamento estabelecido para esta paciente pode ser cogitado para casos semelhantes. Tais medidas poderiam ainda reduzir alguns fatores de riscos cardiovasculares, bem como os sintomas próprios da doença.

Todos os autores declararam não haver qualquer potencial conflito de interesses referente a este artigo.
Paulo, 2001. Tese (doutorado) - faculdade de medicina, Universidade de São Paulo.

8. Kisner C, Colby L. Exercícios terapêuticos, fundamentos e técnicas. São Paulo: Manole, 1998;25-54.

9. Regenga M. Fisioterapia em cardiologia da UTI à reabilitação. São Paulo: Roca, 2000;248-60.

10. Bath P, Less K. A B C of arterial and venous disease. Acute stroke. BMJ 2000;320:920-4.

11. Silva DK, Nahas MV. Prescrição de exercícios físicos para pessoas com doença vascular periférica. Rev Bras Cien Mov 2002;10:55-61.

12. Foss M, Keteyian S. Bases fisiológicas do exercício e do esporte. 6a ed., Rio de Janeiro: Guanabara Koogan, 2000;178-94.

13. Froelicher V, Myers J. Exercício e o coração. 3a ed., Rio de Janeiro: Revinter, 1999;361-73.

14. Hambrecht R, Wolf A. Effect of exercise on coronary endothelial function in patients with coronary artery disease. N Engl J Med 2000;342:454-61. 
15. Froelicher V, Jensen D. Cardiac rehabilitation: evidence for improvement in myocardial perfusion and function. Arch Phys Med Rehabil 1980; 61:517-22.

16. Eckstein RW. Effect of exercise and coronary artery narrowing on coronary collateral circulation. Circ Res 1957;5:230.

17. Bruton A. Muscle plasticity: response to training detraining. Physiotherapy 2002;88:398-409.

18. Pickering J. Tratamento das doenças vasculares periféricas. In: Downie P. Fisioterapia nas enfermidades cardíacas, torácicas e vasculares. $3^{a}$ ed., São Paulo: Panamericana, 1987;335-44.

19. Matheson LM, Isernhagen SJ, Hart DL. Relationships among lifting ability, grip force, and return to work. Phys Ther 2002;82:249-56.

20. Domenico G, Wood E. Técnicas de massagem de Beard. 4aed., São Paulo: Manole, 1998;55-71.

21. American College of Sports Medicine. The recommended quantity and quality of exercise for developing and maintaining cardiorespiratory and muscular fitness in healthy adults. Med Sci Sports Exerc 1990;22:265-74.

22. Benetti M. Condicionamento físico aplicado a doenças cardiovasculares. Rev Bras Med Esporte 1997;3:22-6.

23. Hiatt WR, Wolfel EE, Meier RH, Regensteiner JG. Superiority of treadmill walking exercise versus strength training for patients with peripher- al arterial disease: implications for mechanism of training response. Circulation 1994;90:1866-74.

24. Thompson PD, Buchner D, Pina IL, Balady GJ, Willians MA, Marcus $\mathrm{BH}$, et al. Exercise and physical activity in prevention and treatment of atherosclerotic cardiovascular disease. Arterioscler Thromb Vasc Biol 2003;23:E42-51.

25. McDermott MM, Greenland P, Liu K, Guralnik JM, Celic L, Criqui M, et al. The ankle brachial index is associated with leg function and physical activity: the walking and leg circulation study. Ann Intern Med 2002; 136:873-83.

26. Burns P, Gough S, Bradbury A. Management of peripheral arterial disease in primary care. BMJ 2003;326:584-9.

27. Lakka T, Laukkanen J, Rauramaa R. Cardiorespiratory fitness and progression of carotid atherosclerosis in middle-aged men. Ann Intern Med 2001;134:12-20.

28. Chandler WL, Schwartz RS, Stratton IR, Vitiello MV. Effects of endurance training on the circadian rhythm of fibrinolysis in mem and women. Med Sci Sports Exerc 1996;28:647-58.

29. Jen $\mathrm{C}, \mathrm{Chan} \mathrm{H}$, Chen H. Chronic exercise improves endothelial calcium signaling and vasodilatation in hypercholesterolemic rabbit femoral artery. Arterioscler Thromb Vasc Biol 2002;22:1219-24. 